\title{
Growth of adenoid cystic carcinoma of the parotid during pregnancy: A case report
}

\author{
Joel Davies, Gian-Marco Busato, Antoine Eskander and Kevin Higgins* \\ Department of Otolaryngology - Head \& Neck Surgery/Surgical Oncology, Sunnybrook Health Science Centre, University of Toronto, Toronto, Ontario, Canada
}

\begin{abstract}
Background: The association between pregnancy and growth of salivary gland malignancies has not been well elucidated due to the paucity of reports in the literature. Adenoid cystic carcinoma of the head and neck is known to share histologic characteristics with adenocarcinomas of the breast. A small number of studies have found varying degrees of positive expression of both estrogen and progresterone receptors within adenoid cystic carcinoma. While a few case reports have observed a link between pregnancy and salivary gland tumour growth, none have reported on growth of adenoid cystic carcinoma of the parotid gland under such circumstances.

Case presentation: A 31-year-old Chinese woman presented with growth of a right parotid lesion during pregnancy that was associated with facial nerve palsy and neuropathy of the post-auricular skin. On MRI, the mass was adjacent to the stylomastoid foramen and there was evidence of perineural invasion of the facial and greater auricular nerves. A fine needle aspirate biopsy confirmed adenoid cystic carcinoma. She underwent right radical parotidectomy, ipsilateral neck dissection, temporal bone resection and primary neurorrhaphy of facial nerve branches with adjuvant radiotherapy.

Conclusion: This article represents the third reported case of salivary gland malignancy growth during pregnancy and the first case of adenoid cystic carcinoma. With a focus towards adding to a body of literature examining an association between pregnancy and salivary gland tumors, our report may stimulate interest in better understanding the complex biological behavior of such tumors.
\end{abstract}

\section{Background}

Adenoid cystic carcinoma represents an uncommon head and neck malignancy, accounting for approximately $1 \%$ of all cancers of the head and neck [1]. Of all parotid gland malignancies, the incidence of adenoid cystic carcinoma is between $10-30 \%[2,3]$. This tumor has been known to have similar histology to adenocarcinomas of the breast and, in some cases, can be indistinguishable [3]. While the role of pregnancy related hormones on growth of salivary gland malignancies has yet to be fully elucidated, there is some evidence that estrogen, progesterone or other pregnancy related hormones may play a role [3-6]. The few studies that have examined the role of estrogen and progesterone receptors in adenoid cystic carcinoma using immunohistochemistry have demonstrated varying degrees of positive expression [3,5]. Despite this, only a few case reports have linked pregnancy to salivary gland tumour growth, and none have reported on adenoid cystic carcinoma of the parotid gland.

To date, two other case reports have documented growth of a salivary gland malignancy during pregnancy. Arai, et al. reported a case of a 32-year-old Japanese woman who presented in the third trimester of pregnancy with growth of a right neck mass in the parotid region over a three-month period [7]. Initial fine needle aspirate biopsy failed to demonstrated evidence of malignancy. However, one year following delivery, she underwent total parotidectomy with preservation of the facial nerve after an intraoperative frozen section demonstrated malignant cells. The final pathologic diagnosis was a rare low grade cribriform cystadenocarcinoma [7]. Al-Zaher and Obeid reported on a 25-year-old woman from United Arab Emirates who presented in the second trimester of pregnancy with a right parotid mass confirmed as acinic cell carcinoma on cytologic studies. She underwent superficial parotidectomy with sparing of the facial nerve. She developed a recurrence four years later during the third trimester of her second pregnancy. She subsequently underwent revision total parotidectomy, ipsilateral neck dissection and adjuvant external-beam radiation therapy [8]. Herein we discuss the third reported case of rapid growth of a salivary gland malignancy during pregnancy and the first case of adenoid cystic carcinoma.

\section{Case Report}

A 31-year-old female of Chinese descent was referred for evaluation of a right neck mass. This mass had been observed by her family doctor for several years and noted to be small and stable in size. However, during the third trimester of her first pregnancy, rapid growth and onset of neuropathic pain along the post-auricular skin in the greater auricular nerve distribution was noted. She had no history of head and neck radiation treatment or a personal or family history of salivary neoplasms. A firm and non-mobile mass was observed to be firmly planted between the mastoid tip and angle of the mandible. At the time of initial assessment, she was noted to have intact facial nerve function and no overlying skin changes. The remainder of her examination was unremarkable. A bedside ultrasonography examination was performed with a $12 \mathrm{MHz}$ linear array probe that demonstrated a lobulated

*Correspondence to: Kevin Higgins, Sunnybrook Health Sciences Centre, 2075 Bayview Avenue, Room M1 102, Toronto, ON, M4N 3M5, Canada, E-mail: Kevin.Higgins@sunnybrook.ca

Key words: parotid neoplasm, adenoid cystic carcinoma, pregnancy, radical parotidectomy, facial nerve reconstruction

Received: February 05, 2019; Accepted: February 12, 2019; Published: February 15,2019 
hypoechoic lesion between the mastoid tip and angle of mandible measuring 2.0 X 2.0 X $2.7 \mathrm{~cm}$ (Figure 1). Ultrasound-guided fine needle aspirate biopsy demonstrated cytology consistent with adenoid cystic carcinoma.

During close follow-up in the course of her pregnancy, she was observed to have mild facial nerve impairment (House Brackmann Grade 2) and worsening neuropathic pain in the greater auricular nerve distribution. She underwent further radiologic evaluation with CT and MRI which revealed enhancement of the facial nerve at the stylomastoid foramen and greater auricular nerve on MRI consistent with perineural spread (Figure 2). There were no distant metastases or contraindications for surgical treatment based on cross sectional imaging.

Following a discussion with the multidisciplinary tumor board, a right radical parotidectomy, ipsilateral selective neck dissection (levels II/III and VA) with possible temporal bone resection, facial nerve sacrifice and nerve grafting was recommended. During the procedure, frozen section pathology confirmed invasion of the facial nerve at the level of the stylomastoid foramen (Figure 3). As such, the facial nerve was sacrificed and removed en bloc with the tumor. Temporal bone resection was performed with frozen sectioning of the proximal facial nerve to confirm a negative margin and to have a proximal stump for nerve grafting (Figure 4). Nerve grafting was completed as planned with ansa cervicalis to marginal mandibular branch, masseteric nerve to zygomatic branch and facial nerve mastoid stump to the upper branches of the facial nerve using a cable graft from the nerve to vastus lateralis (Figure 5). The defect was reconstructed with a left anterolateral thigh free flap for volume restoration/coverage and a gold weight was inserted into the upper eyelid to enable eyelid closure.

The final histopathology confirmed the diagnosis of adenoid cystic carcinoma, cribriform subtype, with evidence of perineural and bony invasion at the level of stylomastoid foramen. Additional testing demonstrated that the tumor was estrogen and progesterone receptor negative. All lymph nodes were found to be negative for malignancy. Therefore, following surgery she was staged at pT4aN0M0. At the time of this report, she is in the midst of completing postoperative intensity modulated radiotherapy to a dose of $66 \mathrm{~Gy}$ in 33 fractions. On follow-up clinic visits, there has been evidence of tone within the mid-face suggesting early regeneration of the masseteric to zygomatic neurorrhaphy.

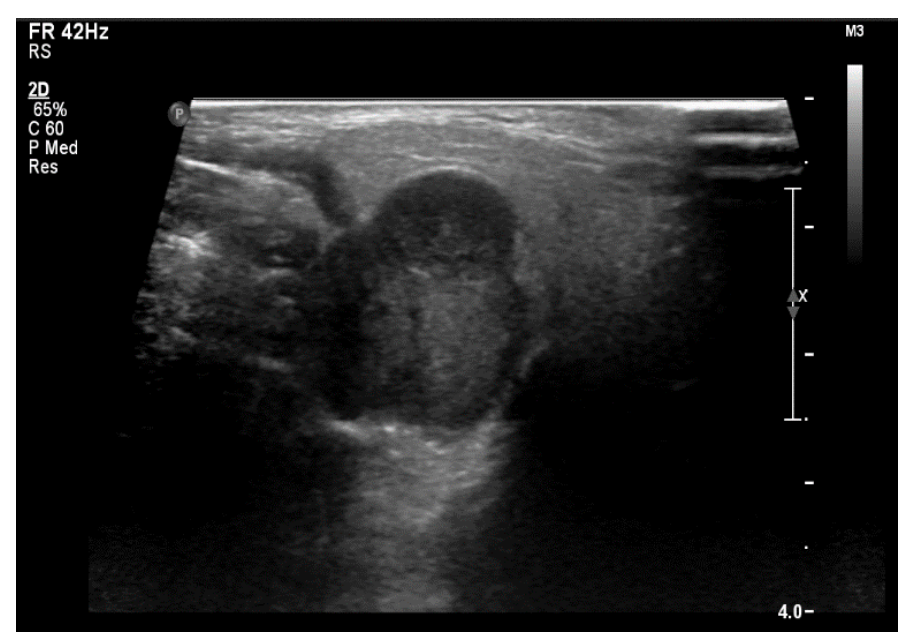

Figure 1. Ultrasound (12 MHz linear array probe) of right parotid mass demonstrating lobulated hypoechoic lesion between the mastoid tip and angle of mandible measuring 2.0 $\mathrm{X} 2.0 \times 2.7 \mathrm{~cm}$ (volume, $5.82 \mathrm{~cm}^{3}$ )

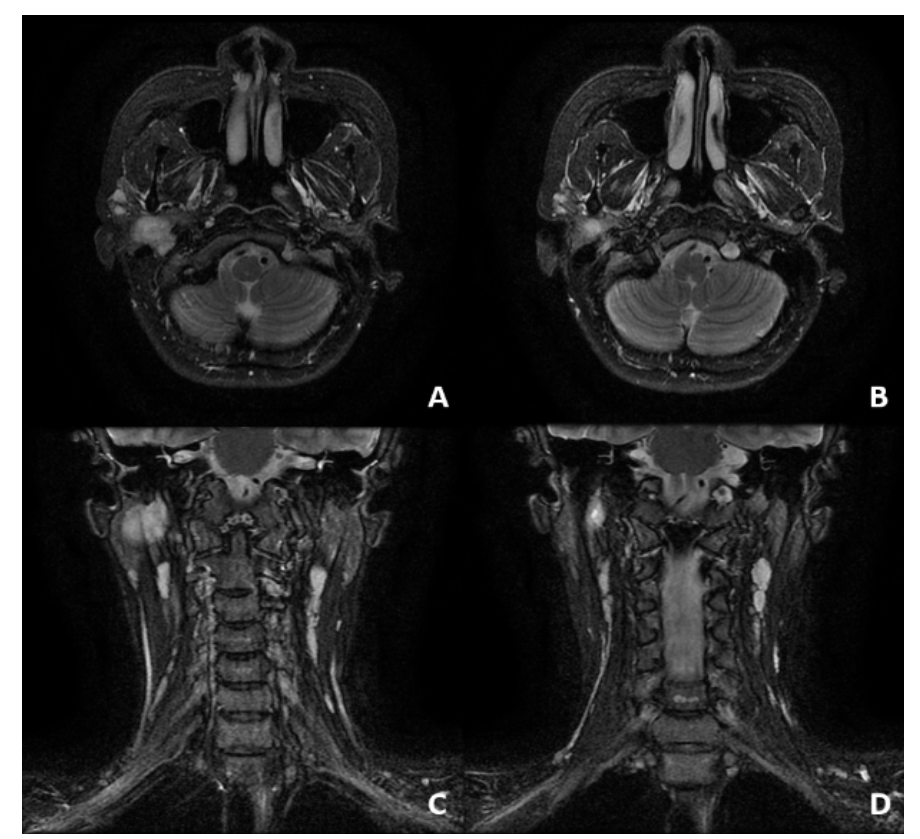

Figure 2. Fat suppressed MRI scans of the head/neck demonstrating right parotid tumor with perineural invasion of facial nerve. (A) axial plane at maximal dimensions of tumor, (B) axial plane of tumor at level of stylomastoid foramen, (C) coronal plane at maximal dimensions of tumor, (D) coronal plane of tumor at level of stylomastoid foramen

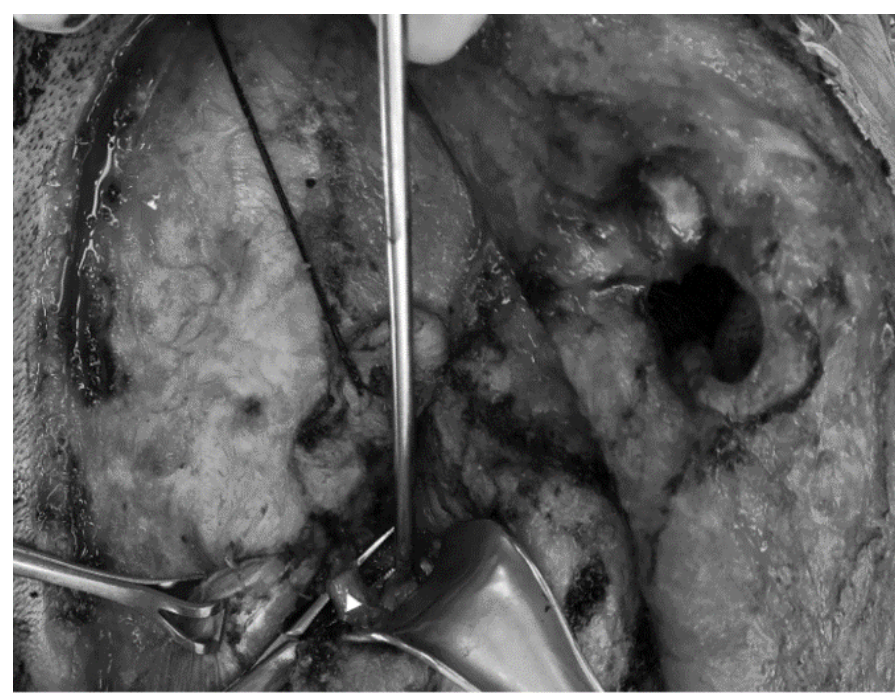

Figure 3. Post-auricular flap exposure and dissection of facial nerve root (white triangle) adherent to parotid tumor at the level of the stylomastoid foramen. Thickened appearance of facial nerve indicative of invasion

\section{Discussion}

The most common presentation of adenoid cystic carcinoma of the salivary glands is that of a slow growing mass, characterized by perineural invasion and often aggressive long-term behavior. Persistence or recurrence of disease and late onset distant metastases is frequently observed. Given the known aggressive potential, adenoid cystic carcinoma is treated as a high-grade neoplasm and, therefore, treated with radical surgical resection and adjuvant radiotherapy. Generally, it is recommended that patients undergo selective neck dissection only in cases of clinically positive neck lymphadenopathy. Historically, incidence rates of occult nodal metastasis are less than $10 \%[4,9]$. 


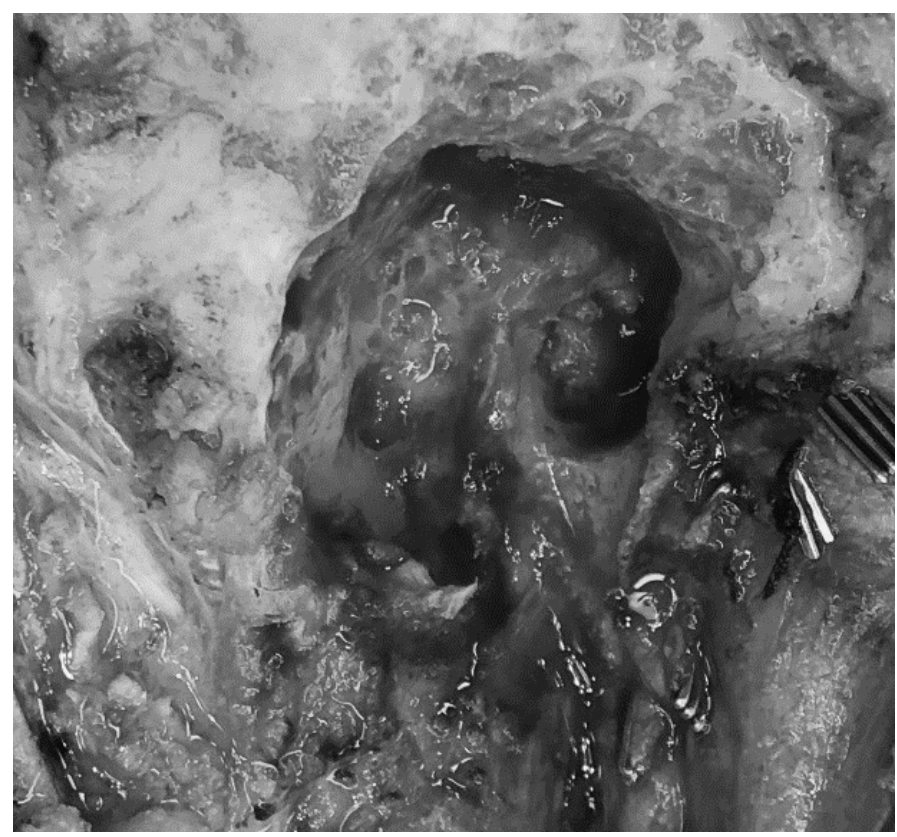

Figure 4. Proximal facial nerve stump (white triangle) within mastoid cavity following temporal bone resection

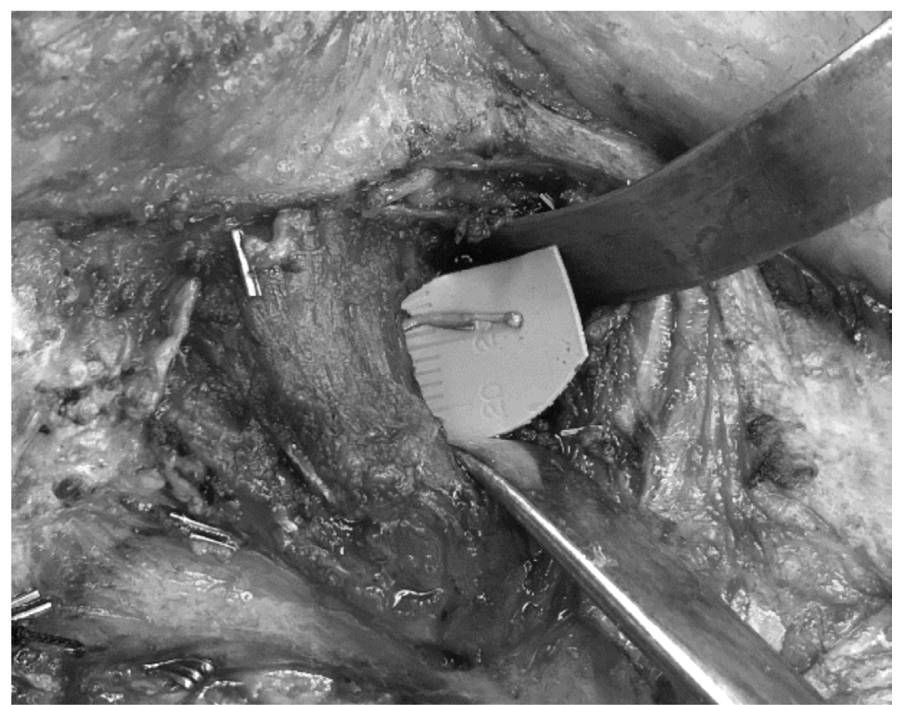

Figure 5. Dissection of distal masseteric nerve through fibres of masseter prior to primary neurorrhaphy to the zygomatic branch of facial nerve

In the case we have presented in this report, the parotid mass had remained stable for several years prior to rapid growth that was associated with pregnancy. Overall, most literature suggests that adenoid cystic carcinoma is observed to have a higher rate of incidence in females for reasons yet unknown [10,11]. Although both estrogen and progesterone receptors have been reported to be expressed by adenoid cystic carcinoma of the salivary glands, this was not observed in our case. Expression of estrogen receptors in adenoid cystic carcinoma specimens varies widely in the literature from $17-92 \%[1,10,11]$. In one case study, tamoxifen therapy was found to provide partial remission in a patient with recurrent metastatic disease [11]. While progesterone is less often found to be expressed in specimens, one study found that $8.5 \%$ of 47 cases reviewed had tumors that stained for progesterone receptors [1]. Given that the histopathology of adenoid cystic carcinoma of the salivary glands is largely indiscernible from sites such as the breast and vulva, growth during pregnancy due to fluctuations in estrogen and progesterone levels is an attractive hypothesis [4]. In this era of personalized cancer therapy, one also wonders if in patients with metastatic disease whether a trial of monoclonal antibody hormone blocking targeted therapy could suppress further disease progression as has been demonstrated in other head and neck cancer sites [12-14].

As a rare form of malignancy, efforts should be put towards better understanding the biology of salivary gland cancers and, more specifically, adenoid cystic carcinoma. The primary observation from this case report, that growth of a salivary gland carcinoma had occurred during pregnancy, is in concordance with other case reports and may provide impetus for other authors to report such cases in the future.

\section{Conclusion}

As the third reported case of growth of a salivary gland malignancy during pregnancy, and the first report of adenoid cystic carcinoma, this report adds to a body of literature that may assist in better understanding the pathophysiologic, and/or genetic, mechanisms behind these rare tumors. Ultimately, this may aid in management through targeted therapy, control of disease progression, and potentially even prevention.

\section{References}

1. Coca-Pelaz A, Rodrigo JP, Bradley PJ, Vander Poorten P, Triantafyllou A, et al. (2015) Adenoid cystic carcinoma of the head and neck - An update. Oral Oncol 51: 652-661.

2. Al-Mamgani A, van Rooij P, Sewnaik A, Sewnaik A, Tans L, et al. (2012) Adenoid cystic carcinoma of parotid gland treated with surgery and radiotherapy: Long-term outcomes, QoL assessment and review of the literature. Oral Oncol 48: 278-283.

3. Eskander A, Irish J, Freeman J, Gullane P, Gilbert R, et al. (2014) Overview of major salivary gland cancer surgery in Ontario (2003-2010). J Otolaryngol Head Neck Surg 43: 50. [Crossref]

4. Barrera JE, Shroyer KR, Said S, Hoernig G, Melrose R, et al. (2008) Estrogen and progesterone receptor and p53 gene expression in adenoid cystic cancer. Head Neck Pathol 2: 13-18. [Crossref]

5. Mujtaba H, Atique M, Batool I, Farooq Umer M (2017) Immunohistochemical evaluation of oestrogen receptors in adenoid cystic carcinoma of salivary gland. J Ayub Med Cell Abottabad 29: 535-539.

6. Dori S, Trougouboff P, David R, Buchner A (2000) Immunohistochemical evaluation of estrogen and progesterone receptors in adenoid cystic carcinoma of salivary gland origin. Oral Oncol 26: 450-453.

7. Arai A, Taki M, Mimaki S, Ueda M, Hori S (2009) Low-grade cribriform cystadenocarcinoma of the parotid gland: A case report. Auris Nasus Larynx 36: 725-728.

8. Al-Zaher NN, Obeid AA (2011) Acinic cell carcinoma in pregnancy: a case report and review of the literature. J Med Case Reports 5:91. [Crossref]

9. Min R, Siyi L, Wenjun Y, Ow A, Lizheng W, et al. (2012) Salivary gland adenoid cystic carcinoma with cervical lymph node metastasis: a preliminary study of 62 cases. Int J Oral Maxillofac Surg 41: 952-957.

10. Rapidis AD, Givalos N, Gakiopoulou H, Faratzis G, Stavrianos SD, et al. (2005) Adenoid cystic carcinoma of the head and neck, Clinicopathological analysis of 23 patients and review of the literature. Oral Oncol 41: 328-335.

11. Eveson JW, Cawson RA (1985) Tumours of the minor (oropharyngeal) salivary glands A demographic study of 336 cases. J Oral Pathol 14: 500-509. [Crossref]

12. Dimery IW, Jones LA, Verjan RP, Raymond AK, Goepfert H, et al. (1987) Estrogen receptors in normal salivary gland and salivary gland carcinoma. Arch Otolaryngol Head Neck Surg 113: 1082-1085. [Crossref]

13. Shadaba A, Gaze MN, Grant HR (1997) The response of adenoid cystic carcinoma to tamoxifen. J Laryngol Otol 111: 1186-1189.

14. Seim NB, Kang SY, Bhandari M, Jones RG, Taknos TN (2017) Personalized medicine approach for an exceptional response to multiple-recurrent and metastatic HER-2 positive oropharyngeal squamous cell carcinoma. Ann Otol Rhinol Laryngol 126: 334-339.

Copyright: (C2019 Davies J. This is an open-access article distributed under the terms of the Creative Commons Attribution License, which permits unrestricted use, distribution, and reproduction in any medium, provided the original author and source are credited. 